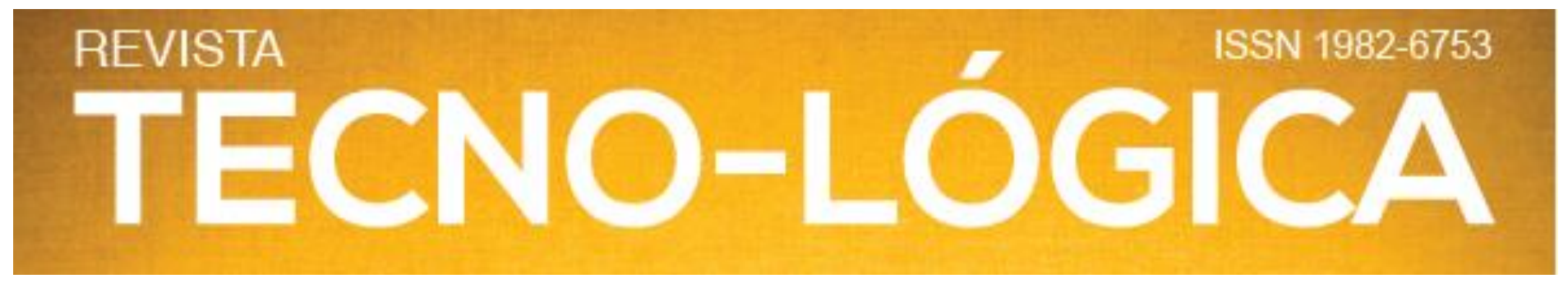

\title{
VALIDAÇÃO DE UM PROTÓTIPO DE ANEMOSCÓPIO BASEADO EM UM MAGNETÔMETRO POR MEIO DA COMPARAÇÃO COM UMA ESTAÇÃO AUTOMÁTICA VAISALA MODELO WXT510
}

\author{
Sergio Roberto Sanches*, Cássio Aurélio Suski, Mario Francisco Leal de Quadro
}

Instituto Federal de Santa Catarina, 88307-303, Itajaí, Brasil.

*E-mail: sergio.sanches@ifsc.edu.br

Recebido em: 09/03/2021

Aceito em: 12/07/2021

DOI: $10.17058 /$ tecnolog.v25i2.16297

\section{RESUMO}

Devido a sua alta variabilidade espacial e temporal, a direção do vento é um dos elementos meteorológicos que necessitam precisão e eficiência em sua medida. Este artigo tem por objetivo a validação de um protótipo de anemoscópio construído baseado em um magnetômetro, pela comparação com os dados medidos por uma estação meteorológica portátil marca Vaisala modelo WXT510. O desenvolvimento do protótipo se dividiu em: (i) fabricação do conjunto mecânico, (ii) fabricação do conjunto eletrônico; (iii) programação do controlador Arduino e (iv) coleta e tratamento dos dados de direção do vento. A leitura da direção do vento se dá no alinhamento entre o corpo móvel com o ímã de neodímio embutido e o vento, sendo que o magnetômetro mede o campo magnético e envia os dados para um controlador Arduino, que calcula a direção do campo magnético com os dados obtidos, gravando a média desta direção a cada minuto em um cartão de memórias SD (Secure digital). O protótipo foi colocado em teste em campo ao lado de uma estação portátil, marca Vaisala, modelo WXT510, durante 47 dias (entre agosto e setembro de 2019), com as duas estações.

Palavras-chave: Anemoscópio. Vento. Magnetômetro.

\section{Introdução}

Elementos meteorológicos são variáveis associadas ao tempo e que podem ser medidas por meio de instrumentos adequados e específicos. Dentre estas variáveis pode-se elencar vento, pressão atmosférica, temperatura e umidade relativa do ar como as mais conhecidas. Estas variáveis, em longo prazo, caracterizam o clima de um determinado local, como um histórico do comportamento destes elementos ao longo do tempo. Segundo Whiteman [1] e Li et al [2], o vento é formado pelo movimento do ar causado pelo gradiente horizontal da pressão, sendo que a localização dos centros de alta e baixa pressão é uma característica fundamental nos mapas meteorológicos, pois fornecem informações sobre a direção e a velocidade do vento.

Devido à dinâmica dos centros de alta e baixa pressão em superfície, associados a presença de sistemas meteorológicos, a medição da direção e velocidade do vento ainda é um grande desafio para a meteorologia principalmente devido a influência do relevo, pois este atua como uma barreira para o deslocamento do ar e influencia na medição do vento na região da camada limite planetária (CLP). Nesse sentido, o vento é o mais variável entre todos os elementos meteorológicos. Em determinadas condições meteorológicas, a velocidade do vento aumenta ou diminui pela metade em poucos segundos.

De acordo com a Organização Meteorológica Mundial [3], a medição da direção e velocidade do vento em uma estação meteorológica pode ser aplicada em diversos setores, desde a definição da climatologia de um determinado local, aplicações aeronáuticas ou estudos sobre a dispersão de poluentes. Para cada aplicação tem-se um requisito de intervalos de medição e formas de cálculo de velocidade e direção do vento.

Com relação às propriedades deste elemento meteorológico, de acordo Emeis [4], o vento é um vetor, caracterizado por um módulo (velocidade do vento) e uma direção (direção do vento).

Geralmente, a componente vertical do vento é muito menor que a sua componente horizontal. Portanto, 


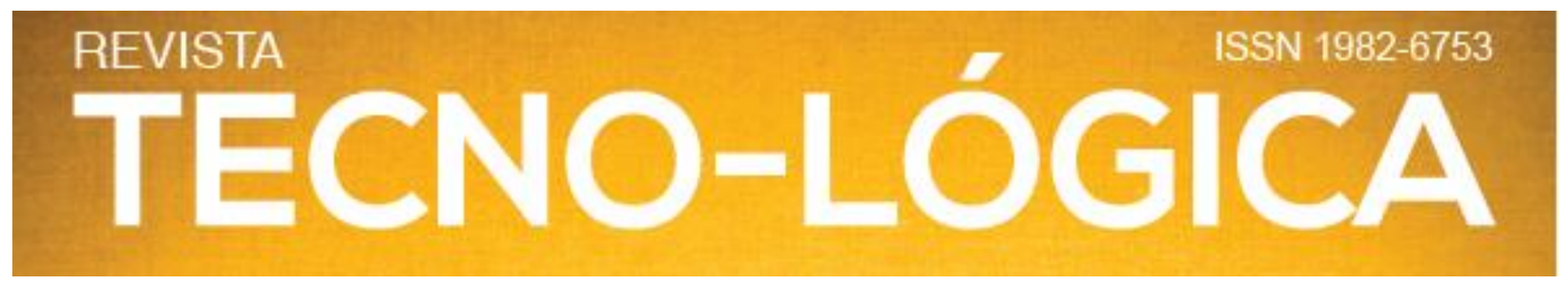

frequentemente, apenas a velocidade horizontal do vento é medida. Burt [5] descreve que a direção do vento se refere à origem do vento, e seu instrumento de medição precisa ser alinhado com precisão ao norte verdadeiro, que é ligeiramente diferente do norte magnético mostrado por uma bússola magnética. Além disso, sua direção pode, ocasionalmente, variar até 180 graus, dentro de um minuto e pode fazer várias voltas ao redor da bússola dentro de uma ou duas horas. Ainda, segundo Khaled e Saif [6] a velocidade do vento depende da localização do instrumento de medição, portanto todos os arredores afetam a velocidade do vento local. Devido a esta influência, torna-se importante levar em conta a orografia nos estudos de medidas anemométricas.

Em combinação com a força de desvio causada pela rotação da Terra (força de Coriolis) e a forma das bacias oceânicas, os ventos determinam o padrão de características do sistema mundial de correntes superficiais. Nesse sentido, o processo de medição das condições meteorológicas se tornou mais fácil em comparação com as décadas anteriores devido a evolução tecnológica [7]. Além disso, segundo Ram e Gupta [8], através do uso de sensores miniaturizados para medir os parâmetros físicos e ambientais e monitorar as condições meteorológicas, os resultados serão mais precisos e todo o sistema será mais rápido, consumindo menos energia.

De acordo com Bae e Mo [9] e Uchida e Kawashima [10] o anemoscópio é um instrumento utilizado para apontar a direção do vento. Quando o vento incide no anemoscópio, ele é estabilizado na sua direção através de oscilações atenuadas. $\mathrm{O}$ formato do anemoscópio baseia-se em um corpo rígido girando em torno de um eixo, e o torque do vento o movimenta alinhando sua direção.

A indicação da direção do vento é lida por meio de um encoder absoluto que faz a leitura da posição do instrumento em relação ao norte verdadeiro, sendo que, o ângulo de $360^{\circ}$ é alinhado com o norte verdadeiro, ficando assim o leste aos $90^{\circ}$, o sul aos $180^{\circ}$, oeste aos $270^{\circ}$, sendo que o ângulo de $0^{\circ}$ somente é computado no caso de ausência de vento.

O magnetômetro é um instrumento para medir a intensidade e a direção de um campo magnético, sendo utilizado na fabricação de bússolas eletrônicas, em celulares e outros aparelhos eletrônicos. Devido à característica de medir a sua orientação em relação ao norte magnético e a facilidade de tratamento destes dados o magnetômetro pode ser utilizado em anemoscópios, como o protótipo desenvolvido por Araki et al. [11], que utilizou um magnetômetro do tipo fluxgate com a finalidade de indicar a direção do vento.

Pelas suas características, o magnetômetro pode proporcionar uma resolução de 360 divisões por volta, consideravelmente maior em relação às estações meteorológicas de baixo custo, como as utilizadas no estudo de Jenkins [12], com a resolução de 8 a 16 divisões por volta, e Li et al. [13], que elaboraram um protótipo com 36 divisões por volta.

Esse artigo tem como objetivo principal a validação de um protótipo de anemoscópio construído com base em um magnetômetro, por meio da comparação com os dados medidos por uma estação meteorológica portátil validada de acordo com as normas da Organização Meteorológica Mundial (OMM).

\section{Parte Experimental ou Metodologia}

\subsection{Desenvolvimento do Anemoscópio}

O desenvolvimento do protótipo se dividiu em fabricação do conjunto mecânico, fabricação do conjunto eletrônico, programação e coleta e tratamento dos dados de direção do vento.

\subsubsection{Conjunto Mecânico}

A mecânica se constituiu na fabricação do anemoscópio, suportes, bases niveladoras e hastes de fixação, utilizando-se de máquinas ferramentas. O desenvolvimento da estrutura mecânica iniciou-se com o projeto, modelagem e simulação de movimentos dos componentes no software de CAD (Computer Assisted Design) Inventor 2018, seguido do detalhamento em desenho técnico dos elementos a serem fabricados. O anemoscópio foi laminado em PRFC (plástico reforçado com fibra de carbono), com a inserção de um ímã de neodímio com diâmetro de $6 \mathrm{~mm}$ e comprimento de $16 \mathrm{~mm}$ no centro de sua tubulação. Na base do corpo do anemoscópio tem-se o alojamento do rolamento, responsável pela rotação livre do anemoscópio. A massa total das partes móveis do anemoscópio ficou em $29 \mathrm{~g}$. A base do anemoscópio foi fabricada em alumínio, fixando-se o módulo magnetômetro HMC5883L com resina epóxi a fim evitar a presença de umidade.

Com o objetivo de minimizar o efeito do atrito na rotação do anemoscópio, bem como evitar o efeito da oxidação de materiais em contato com a umidade, utilizou-se no conjunto um rolamento fabricado em cerâmica. Os moldes utilizados na laminação do corpo do anemoscópio foram fabricados em máquinas ferramentas convencionais e com Comando Numérico Computadorizado (CNC) utilizando programas elaborados manualmente e com auxílio de softwares de CAM (Computer Aided Manufacturing). A base de alinhamento do anemoscópio, além da regulagem de nível, conta ainda com uma regulagem para alinhamento de sua base com os pontos cardeais. $\mathrm{O}$ 


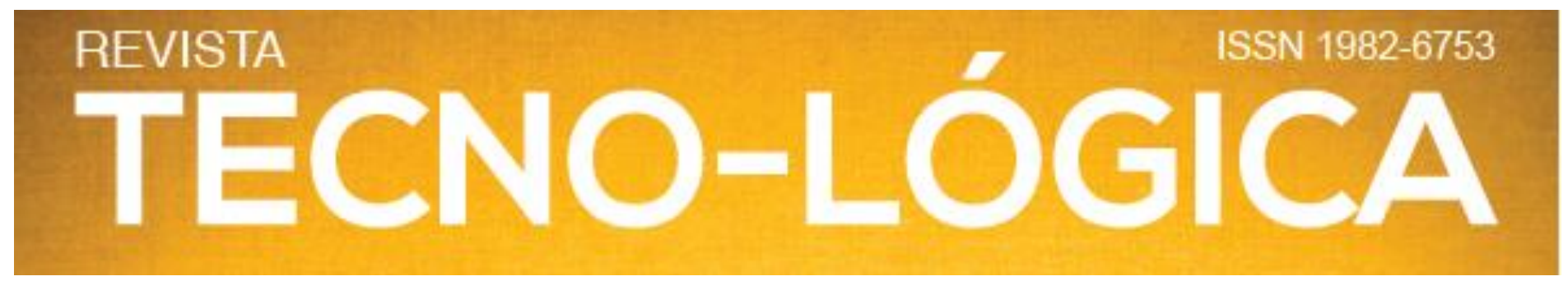

alinhamento horizontal do sensor com a base do anemoscópio foi realizado com o objetivo de interferir o mínimo possível no resultado das medições. A haste para fixação do conjunto ao solo foi fabricada em perfil estrutural de alumínio com seção retangular de 40 x $80 \mathrm{~mm}$, bem como a estrutura de fixação da parte eletrônica.

Por fim, o anemoscópio foi fixado em sua base e, posteriormente, no suporte de alumínio formando outro subconjunto denominado módulo de vento. A comunicação entre os subconjuntos foi executada com um cabo blindado de quatro vias com $10 \mathrm{~m}$ de comprimento e conectores de cinco vias. A figura 1 ilustra as partes do protótipo após a montagem.

A disposição dos cabos de alimentação e transmissão de dados foi projetada com o cuidado necessário para que seu efeito magnético não influencie na medição, mesmo que sua influência seja mínima devido à baixa corrente de alimentação do magnetômetro $(100 \mu \mathrm{A})$.
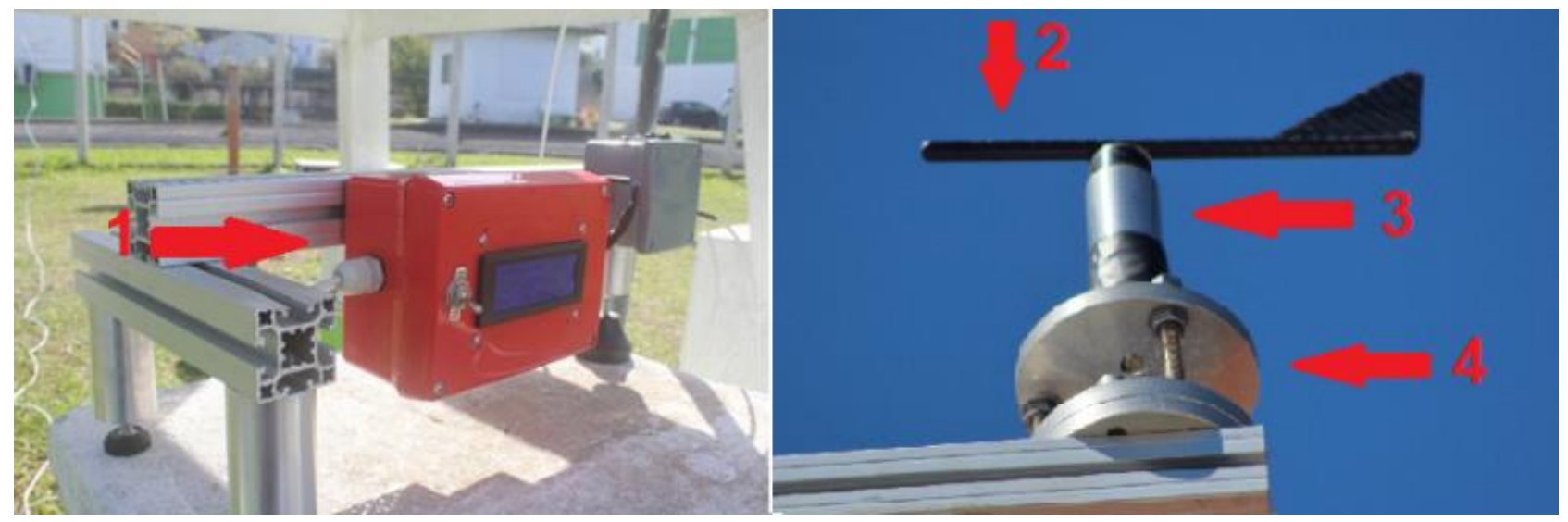

Figura 1 - Partes do protótipo do anemoscópio: (1) Módulo de controle, (2) Corpo do anemoscópio, (3) Suporte do sensor e (4) Base niveladora.

\subsubsection{Conjunto Eletrônico}

Para efetuar a medição da direção do vento, foi escolhido o sensor magnetômetro HMC5883, que registra a intensidade do campo magnético mais próximo na direção dos seus 3 eixos (x, y e $z$ ), e envia sob demanda os valores obtidos pelo sistema de armazenamento por meio da comunicação I2C. A partir dos valores do campo magnético medido calcula-se o ângulo de alinhamento do módulo HMC5883 com os pólos do ímã de neodímio.

\subsubsection{Programação}

Devido ao baixo custo e a facilidade em seu uso, o Arduino vem sendo utilizado no desenvolvimento de estações meteorológicas, como a desenvolvida por Farhat et al. [14] que utilizou uma placa do modelo Uno em uma estação medindo elementos meteorológicos em tempo real. Nesse sentido, a programação foi executada levando em consideração as características da direção do vento e dos sensores, bem como as normas elaboradas pela OMM e objetivos do protótipo. A direção do vento foi obtida por meio da comunicação I2C entre o módulo HMC5883 e o Arduino. A leitura da posição do anemoscópio se dá a cada segundo, e consiste em duas variáveis horizontais de intensidade magnética $\mathrm{x}$ e $\mathrm{y}$.

Estes valores são acumulados durante um minuto em duas variáveis e, posteriormente, calculando suas médias. Com estes valores médios de $\mathrm{x}$ e y é calculada a resultante da intensidade magnética que corresponde à direção média do vento no minuto decorrido. Após cada minuto as variáveis de $\mathrm{x}$ e y acumulados são zeradas e a direção média do vento é mostrada no monitor serial da IDE do Arduino.

A cada minuto completo o relógio de tempo real envia um sinal ao Arduino e é realizado o cálculo da direção média do vento. Os dados obtidos são gravados no cartão SD pelo módulo leitor e gravador de cartão micro SD juntamente com a data e o horário. O conjunto eletrônico foi montado em uma caixa plástica, tendo em seu interior o controlador Arduino, relógio de tempo real, leitor e gravador de cartão micro SD, sendo fixada em um suporte de alumínio formando um subconjunto denominado módulo de controle. 


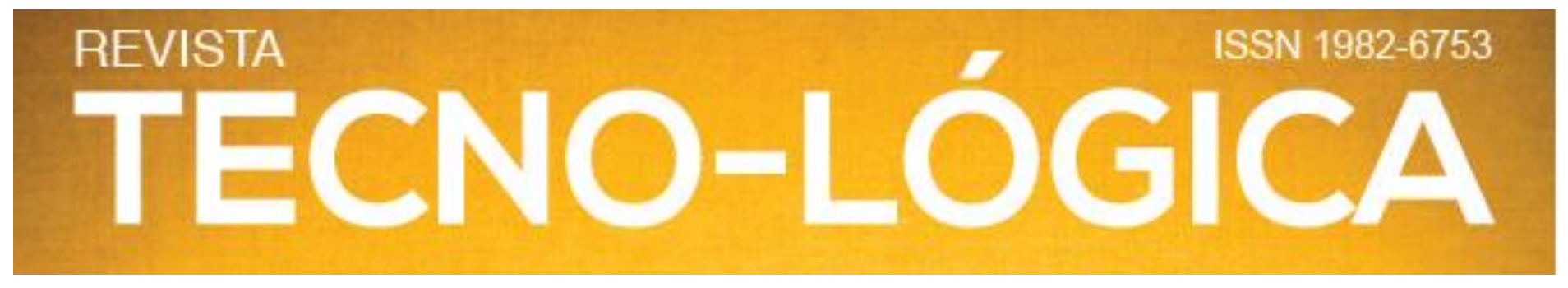

$$
\rho=1-\frac{6 \sum_{i=1}^{n} d^{2}}{n\left(n^{2}-1\right)}
$$

\subsection{Coleta e tratamento dos dados de direção do vento}

A fim de validar a direção do vento medida por um protótipo de anemoscópio baseado em um magnetômetro os dados foram comparados com os obtidos pela estação automática Vaisala, modelo WX510 (número de série $\mathrm{S} / \mathrm{N}$ : H3530006), simultaneamente, conforme localização da figura 2a. O modelo WXT510 além dos elementos meteorológicos direção e velocidade do vento $(\mathrm{m} / \mathrm{s})$, mede a temperatura (C) e umidade relativa do ar (\%), pressão atmosférica (hPa) e precipitação (mm) [10]. Na figura $2 \mathrm{~b}$ observa-se o posicionamento da estação portátil WXT510 (circulada em azul) e do protótipo (circulada em vermelho). A distância horizontal entre elas é de aproximadamente $2 \mathrm{~m}$, e a altura das duas em relação ao solo também é de aproximadamente $2 \mathrm{~m}$.

Pode-se observar também árvores de médio porte ao fundo. Vale salientar que tanto o protótipo como a estação portátil WXT510 foram instalados, durante período de coleta de dados, na localidade da estação meteorológica do Instituto Federal de Santa Catarina (IFSC). Esta estação, apesar de conter os instrumentos convencionais e automáticos, não é considerada como uma estação meteorológica padrão, pois não segue as normas de localização e distância de obstáculos determinadas pela OMM [3].

Foram realizadas 19025 coletas de direção do vento, com registros a cada minuto, durante um período de 47 dias entre 02/08 e 17/09/2019 de forma intermitente. A análise dos dados consistiu em comparar as séries temporais da direção do vento obtida no protótipo com a obtida na estação meteorológica automática Vaisala, modelo WX510, por meio de testes efetuados no software MINITAB. Inicialmente submeteu-se as duas séries ao teste de normalidade de Kolmogorov-Smirnov.

Os testes de normalidade mostraram um resultado de distribuição não normal para as duas séries temporais. Como os testes paramétricos não representam a realidade das amostras, o teste não paramétrico ao qual as amostras foram submetidas foi o coeficiente de Rô de Spearman, que apresenta o grau de associação de duas variáveis medindo a proximidade dos dados, variando entre -1 que indica uma correlação negativa perfeita e 1 uma correlação positiva perfeita, sendo que 0 sugere que não há nenhuma correlação entre as duas séries de dados. O cálculo da correlação de Spearman é realizado através da equação (1). onde: r é o coeficiente de correlação de Spearman, na população da amostra, xi a amostra i da estação WXT510 e yi a amostra i do protótipo.

Devido às características dos dados de direção serem cíclicas, os registros obtidos foram ajustados antes dos testes estatísticos, pois a diferença entre as duas séries temporais é calculada por uma subtração simples. A figura 3 exemplifica o caso de duas direções do vento medidas no quadrante norte. Os vetores n e n' são exemplos de possíveis medições de direção do vento. Do ponto de vista meteorológico a diferença entre eles corresponde ao ângulo $\mathrm{A}$, porém o software estatístico calcula a diferença B. Neste caso foi subtraído $360^{\circ}$ da posição do vetor $\mathrm{n}$, obtendo-se um valor de ângulo negativo, ajustando-se a diferença entre os dois vetores para o ângulo A. Nesse sentido, para realização da medição da direção do vento pelo protótipo (figura 3) foi necessário, primeiramente, desenvolvê-lo com as características necessárias para garantir a eficiência desejada para a mecânica, eletrônica, programação e transmissão dos dados.

\section{Resultados e discussões}

Através da figura 4 pode-se observar a série temporal da direção do vento medida pelas estações WXT510 (Fig. 4a) e pelo protótipo (Fig. 4b) durante a coleta de dados. Foram considerados somente os dados coletados no período de efetivo funcionamento do protótipo.

A distribuição da direção do vento apresentou uma grande variação de amplitude e frequência, possivelmente, intensificada pelos obstáculos físicos como prédios, árvores e morros ao redor da estação WXT510 e do protótipo. Grandes variações na direção do vento, geradas por obstáculos físicos, também foram observadas por Burt [5] e Pannila et al. [15] o qual afirmam que a direção e a velocidade do vento variam continuamente com um período de tempo, mudando a cada minuto, sendo vital a correta exposição dos instrumentos para obter resultados precisos, corretos e comparáveis. E ainda, segundo Khaled e Saif [6], em seu estudo sobre geradores eólicos, a direção do vento pode ser intensamente influenciada pela presença de edificações, árvores e vales. 

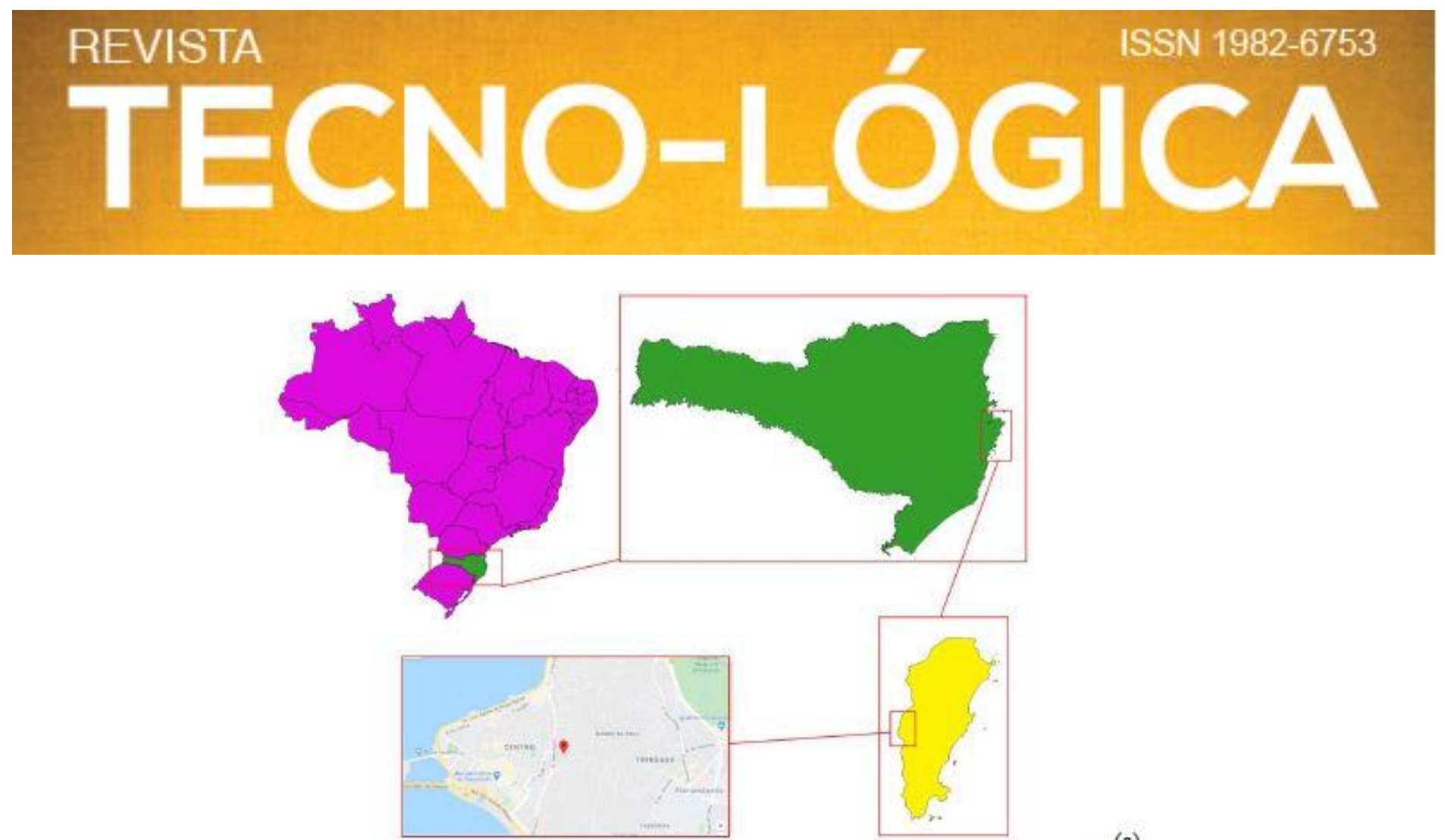

(a)

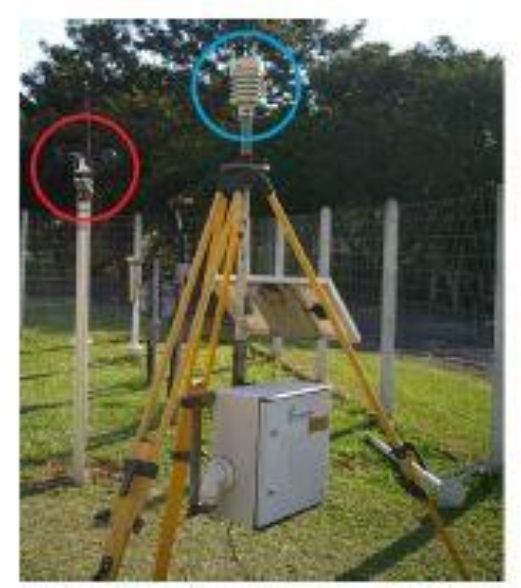

(b)

Figura 2- Localização da (a) instalação das estações meteorológicas na coordenada 27³5'36.1"S 48³2'29.6"O e (b) imagem dos equipamentos com os sensores circulados em azul (WXT510) e vermelho (Protótipo).

No entanto, é possível observar em ambas estações, até o dia 6 de agosto de 2019, o predomínio do vento na direção SE/E, alternando em alguns momentos para o quadrante norte.

Até o dia 13 de agosto de 2019 o vento apresentou predomínio do quadrante norte associado a influência de uma massa de ar quente, responsável pela elevação das temperaturas máximas que atingiram $30^{\circ} \mathrm{C}$ no dia 09 de agosto de 2019. A partir desse dia até o início de setembro, o vento em ambas estações se configurou do quadrante sul, com intervalos curtos de inversão

para o quadrante norte, associado ao ingresso de massas de ar frio. As séries temporais de temperatura (pressão) atmosférica refletiram esse padrão indicando uma redução (aumento) destas variáveis meteorológicas. No final do período do estudo, o vento voltou a ter predomínio do quadrante norte em ambas estações. Esse comportamento similar entre as estações, indica que, visualmente, o protótipo apresentou um comportamento condizente com uma estação certificada. 


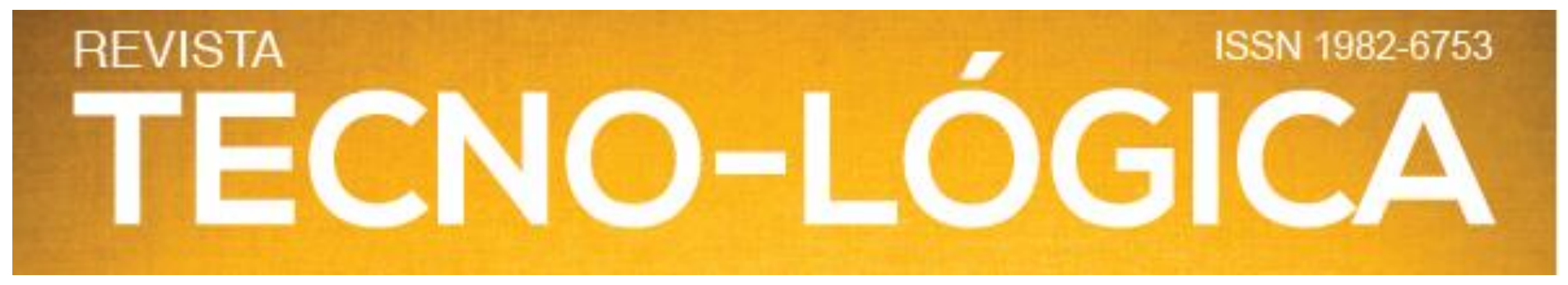

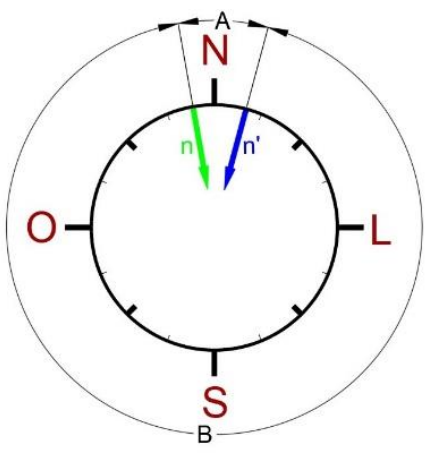

Figura 3 - Exemplo de dois vetores no quadrante norte de direção

Para uma melhor comparação visual entre os dados das duas estações pode-se observar na figura 5 os dados sobrepostos da estação WXT510 e protótipo, no período de 24 horas, assim como a diferença entre as medidas no mesmo período. A análise da figura 5 mostra que a diferença entre a direção do vento medida pelas duas estações se manteve alta, chegando a variações de acima de $100^{\circ}$, tanto positiva, quanto negativa, onde, possivelmente, houve influência dos obstáculos nos arredores do local das estações, causando um fluxo turbulento de ar. Segundo Rodrigues e Pita [16] e Abbasi et al. [17] os escoamentos atmosféricos são de natureza turbulenta, apresentando movimento desordenado com forte interação entre si, não sendo possível uma reprodução experimental, detalhada, dos respectivos campos de velocidades. Outro fato que corrobora essa afirmação é que as maiores diferenças ocorrem no período vespertino, onde o maior aquecimento das superfícies contribui com o aumento do fluxo turbulento.

Dois possíveis agravantes destas discrepâncias encontradas neste estudo são: (i) a distância de cerca de dois metros entre os dois instrumentos de medição adicionados a este fluxo turbulento, e (ii) a diferença entre os métodos de alinhamento das estações com os pontos cardeais, sendo visual na estação meteorológica WXT510 e digital no protótipo.

A figura 6 mostra a distribuição de normalidade das duas séries temporais em todo o período de coleta de dados. Para essa análise realizou-se o teste de normalidade de KolmogorovSmirnov, com nível de significância de 5\%, nas séries temporais obtidas pelo protótipo e pela estação WXT510. Em ambos os casos se obteve $\mathrm{p}<0,010$, ou seja, rejeita-se a hipótese nula de que a distribuição dos dados é normal.

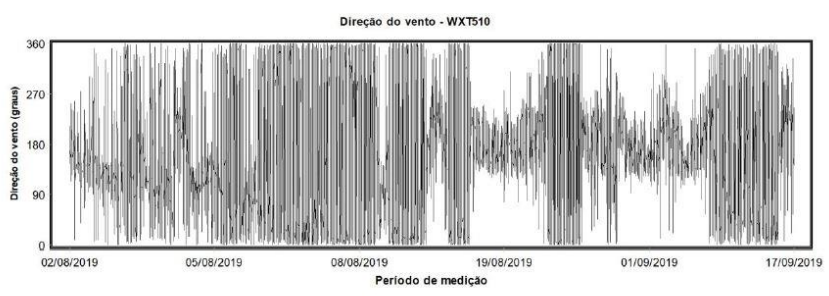

(a)

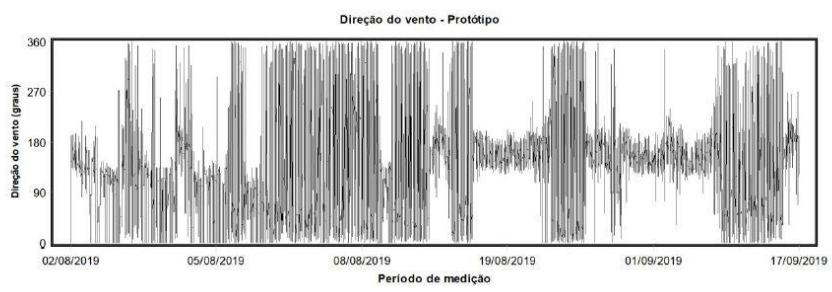

(b)

Figura 4 - Direção do vento (graus) medida pela (a) estação WXT510 e pelo (b) protótipo desenvolvido durante todo o período de coleta de dados.

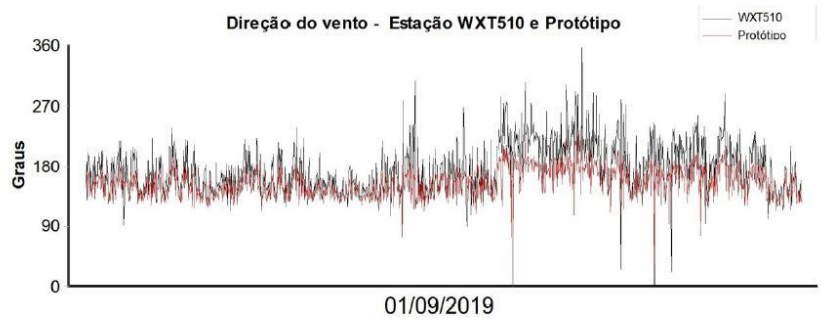

(a)

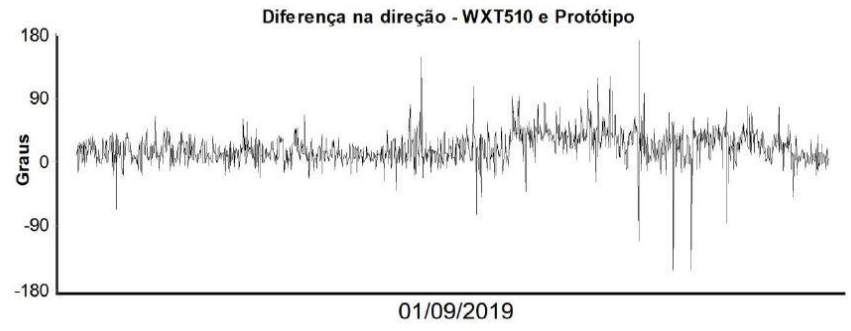

(b)

Figura 5 - Direção do vento (graus) (a) medida pela estação WXT510 (linha preta) e pelo protótipo (linha vermelha) para o período de 24 horas do dia 01/09/2019 e (b) diferença (graus) entre as duas estações. 


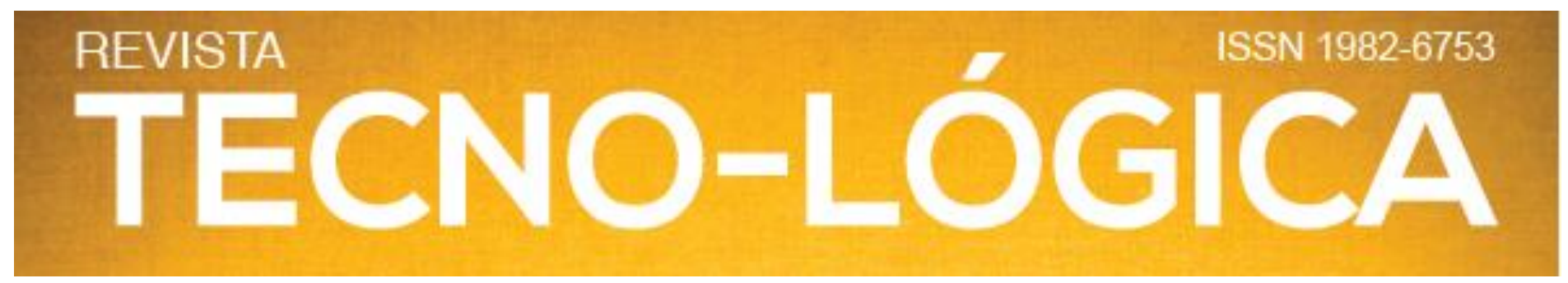

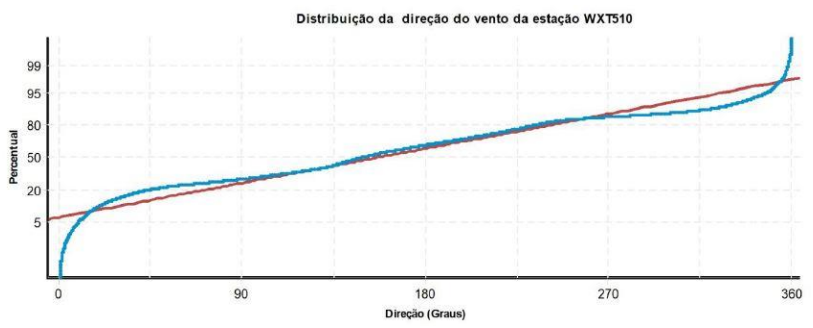

(a)

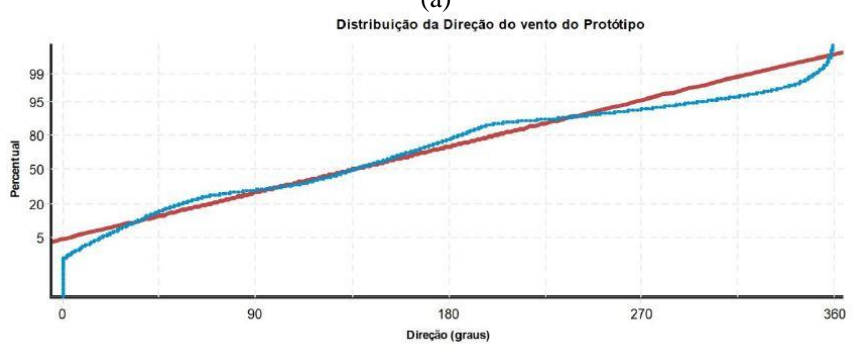

(b)

Figura 6 - Como na figura 4, para a distribuição de frequência da direção do vento.

$\mathrm{Na}$ figura 7 pode-se observar, respectivamente, o histograma da direção do vento determinado na estação WXT510 (Fig. 7a) e no protótipo (Fig. 7b). As linhas vermelhas representam a distribuição normal calculada pelas médias e desvios padrão dos dados e as barras representam a distribuição encontrada em cada série temporal. Nota-se que a distribuição real dos dados não acompanha a distribuição normal, corroborando para o resultado encontrado no teste de normalidade de Kolmogorov-Smirnov. Observa-se também que a estação WXT teve 3 picos de distribuição acima da linha de distribuição normal, enquanto o protótipo tem 2 picos. O protótipo mostra uma quantidade menor de leituras no quadrante oeste (entre 180 e $360^{\circ}$ ) quando comparado à estação WXT510.

Esta, no entanto, teve uma frequência maior de valores medidos entre as direções 90 e $270^{\circ}$. Como a distribuição dos dados não atende ao requisito de normalidade, a média e o desvio padrão utilizados nos testes paramétricos não são estatisticamente relevantes, e desta forma tem-se a necessidade do uso de testes não paramétricos como a correlação de Spearman. Após a correção dos dados a correlação de Spearman entre as duas estações foi calculada em 0,93, indicando que as duas séries são fortemente correlacionadas.

Os valores referentes à média, desvio padrão, população (n) e o valor $\mathrm{p}$ do teste de normalidade de Kolmogorov - Smirnov das séries temporais medidos pela estação WXT510 e pelo protótipo podem ser encontrados na tabela 1 .

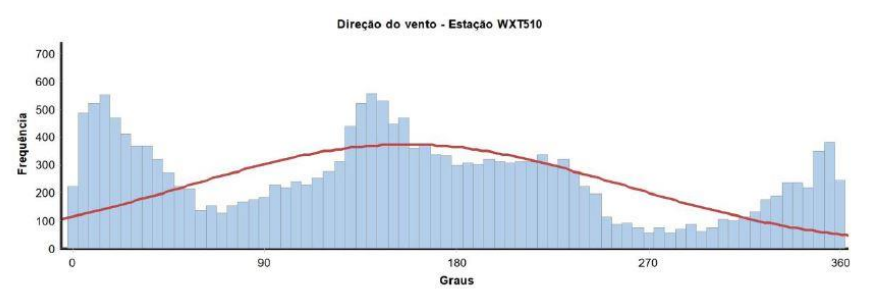

(a)

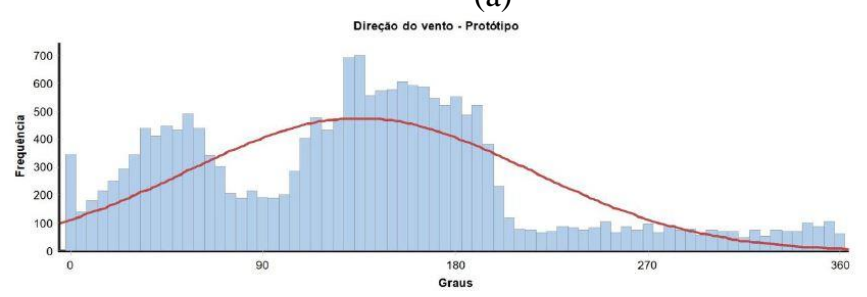

(b)

Figura 7 - Como na figura 4, para o histograma da direção do vento.

Tabela 1. Parâmetros estatísticos avaliados entre as duas estações meteorológicas utilizadas no estudo.

\begin{tabular}{ccl}
\hline Parâmetro & WXT510 & Protótipo \\
\hline Média $\left({ }^{\circ}\right)$ & 156,4 & 135,1 \\
Desvio Padrão & 102,1 & 79,91 \\
População $(\mathbf{n})$ & 19160 & 19025 \\
Valor p & $<0,010$ & $<0,010$ \\
\hline
\end{tabular}

A análise da tabela 1 mostra que o comportamento médio da direção do vento, durante o período do estudo ( 6 a 13 de agosto de 2019), foi de SE, resultado da atuação dos sistemas meteorológicos descritos na figura 4. Comparando os dois sensores observa-se uma diferença na direção média de $21,3^{\circ}$, que pode ser resultado do posicionamento das estações em relação ao norte verdadeiro. Além disso, essa diferença corresponde a 20,8\% do desvio padrão da estação WXT e $26,6 \%$ do desvio padrão do protótipo. O valor elevado do desvio padrão confirma a grande variabilidade das direções dos ventos durante a coleta de dados oriunda dos vários sistemas ocorridos, sobretudo a presença de massas de ar quente, com predominância dos ventos do quadrante norte e massas de ar frio, com ventos predominantemente no quadrante sul. Por fim, a diferença de menos de $0,7 \%$ na população da amostra não impactou nos resultados de forma significativa, mantendo uma forte correlação entre as duas amostras. 


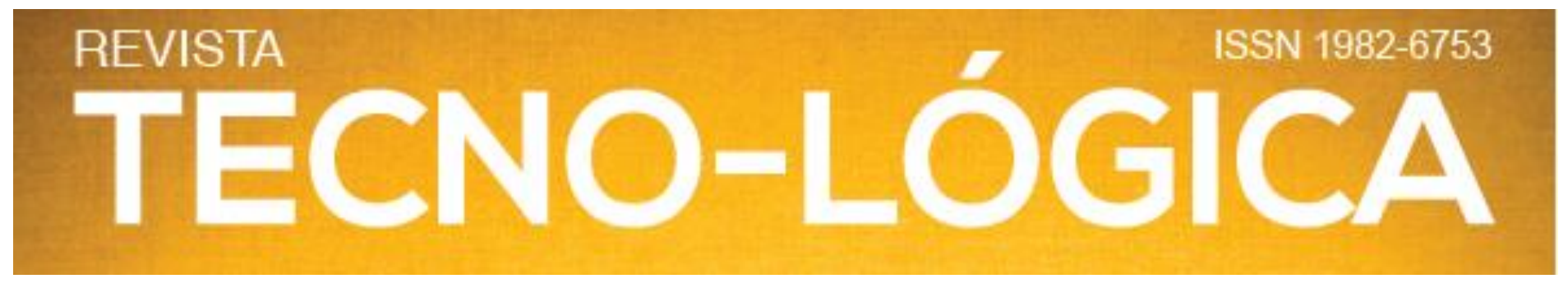

\section{Conclusões}

Neste artigo a validação de um protótipo de anemoscópio construído com base em um magnetômetro, por meio da comparação com os dados medidos por uma estação meteorológica portátil marca Vaisala modelo WXT510 foi estudada. Baseados nos resultados, pode-se obter algumas conclusões que abrangem a construção do protótipo, como também da análise estatística realizada.

Referente a construção do equipamento, verificou-se que uso do magnetômetro em anemoscópios apresentou robustez e confiabilidade na medição da direção do vento, mesmo com a massa total das partes móveis com $29 \mathrm{~g}$. Isso se deve às conchas construídas com plástico reforçado com fibra de carbono, inserção de um ímã de neodímio no centro de sua tubulação e o alojamento do rolamento, fabricado em cerâmica para evitar oxidação, na base do corpo do protótipo, responsável pela rotação livre do anemoscópio. Esse método tornou o conjunto mecânico resistente e sensível às menores variações de direção do vento.

O controlador Arduino necessitou de ajustes na sua programação durante a coleta de dados para evitar-se o seu travamento e pane. Após estes ajustes o controlador funcionou de forma contínua e estável.

O método de alinhamento eletrônico com o Norte, tanto o magnético quanto o geográfico se mostrou prático e com erros mínimos de alinhamento, podendo-se considerar a declinação magnética do local sem maiores dificuldades. Com relação a transmissão dos dados, o baixo consumo do magnetômetro e do controlador Arduino tornam muito vantajoso o uso do equipamento em campo com alimentação por placas solares e baterias. No entanto, ressalta-se que o equipamento pode ser aprimorado com o acréscimo de uma blindagem magnética e posicionamento mais preciso no equipamento, possibilitando a construção de um instrumento de grande confiabilidade.

A análise estatística mostra que o alto índice da correlação de Spearman entre os dados do protótipo e da estação meteorológica portátil WXT510 leva a uma boa confiabilidade dos dados, tornando o protótipo uma opção de baixo custo para aplicações que exigem menor precisão dos dados de direção do vento.

\section{ANEMOSCOPE PROTOTYPE BASED ON A MAGNETOMETER THROUGH COMPARISON WITH A VAISALA AUTOMATIC STATION MODEL WXT510}

ABSTRACT: Due to its high spatial and temporal variability, the wind direction is one of the meteorological elements that need precision and efficiency in its measurement. This article aims to validate an anemoscope prototype built based on a magnetometer, by comparison with data measured by a portable weather station brand Vaisala model WXT510. The development of the prototype was divided into: (i) manufacture of the mechanical assembly, (ii) manufacture of the electronic assembly; (iii) programming the Arduino controller and (iv) collecting and processing wind direction data. The reading of the wind direction occurs in the alignment between the mobile body with the built-in neodymium magnet and the wind, with the magnetometer measuring the magnetic field and sending the data to an Arduino controller, which calculates the direction of the magnetic field with the data obtained, recording the average of this direction every minute on an SD (Secure digital) memory card. The prototype was put to a field test next to a portable station, brand Vaisala, model WXT510, for 47 days (between August and September 2019), with the two stations recording the wind direction data every minute.

Keywords: Anemoscope. Wind. Magnetometer.

\section{Referências}

[1] WHITEMAN, C. D. Mountain Meteorology : Fundamentals and Applications. Oxford University Press, New York, 2000.

[2] LI J., WANG W.,LU J.,YUE J., BURNS A. G., YUAN T., CHEN X., DONG W. A Modeling Study of the Responses of Mesosphere and Lower Thermosphere Winds to Geomagnetic Storms at Middle Latitudes. Journal of Geophysical Research: Space Physics Volume 124, Issue 5 p. 3666-3680. 2019

[3] WMO. Guide to Meteorological Instruments and Methods of Observation Volume I -Measurement of Meteorological Variables. World Meteorological Organization, No. 8, Switzerland, 2018.

[4] EMEIS, S. Wind Energy Meteoroloy: Atmospheric Physics for Wind Power Generation. Springer international Publishing, 2a edição, Alemanha, 251p. 2018.

[5] BURT, S. The Weather Observer's Handbook. New York: Cambridge University Press, 2012.

[6] KHALED, M. D.; SAIF, M. B. R. Low Cost High Altitude Automatic Weather Station Design. Solar Energy and Sustainable Development, Vol. 7, Líbia, 12p. 2018 .

[7] NASR, M.S.; ALEBADY, W.Y.Y. Design and Implementation of a Smart Weather Station Based on Internet of Things. Journal of Babylon University Engineering Sciences, $\mathrm{n}^{\circ}$ 5/Vol.25: Iraque, 2017.

[8] RAM, K.S.S.; GUPTA, A.N.P.S. IoT based Data Logger System for weather monitoring using Wireless sensor networks. International Journal of Engineering Trends and Technology (IJETT), Volume 32 Numero 2- Índia, 2016.

[9] BAE, S.; MO, D. Y. Dynamical analysis of an anemoscope in the phase plane. Applied Mathematical Modelling, Vol.34(7), pp.1884-1891, República Popular Democrática da Coreia, 8p. 2010.

[10] UCHIDA, T.; KAWASHIMA, Y. New Assessment Scales for Evaluating the Degree of Risk of Wind Turbine Blade Damage Caused by Terrain-Induced Turbulence. Energies,12, 2624,2019

[11] ARAKI, T.; IMAMURA, S.; RIKIISHI, K.; ISHIDA, S. An application of the Flux-Gate Magnetometer to measuring the wind direction. IEEJ Transactions on Sensors and Micromachines, ${ }^{\circ}$ 122, Japão, 2002. 


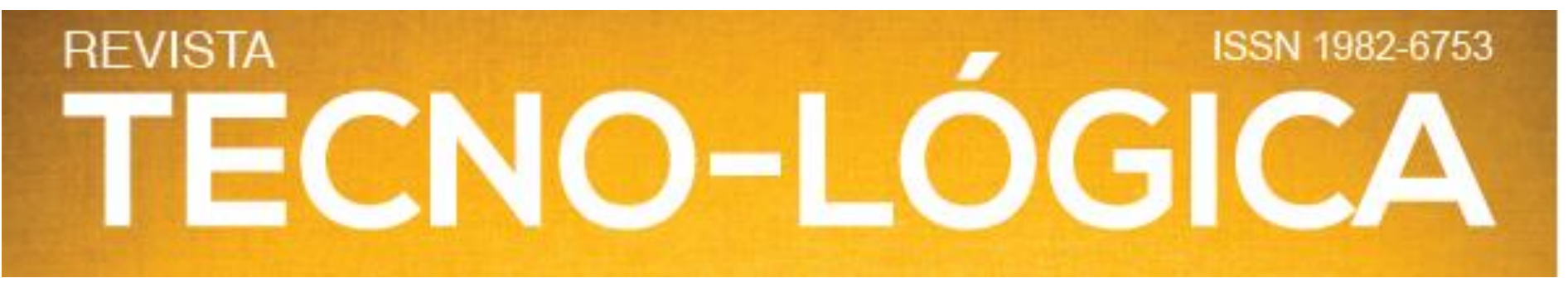

[12] JENKINS G. A comparison between two types of widely used weather stations. Royal Meteorological Society - Weather, vol. 69, n, USA, 2014

[13] Li X., Zhang Z., Li L.; "Wind direction sensing system based on fiber Bragg grating sensor," Applied Optics. 56, 9862-9867 (2017)

[14] FARHAT, M.; ABDUL-NIBY, M.; ABDULLAH, M.; NAZZAL, A. A. Low

Cost Automated Weather Station for Real Time Local Measurements. Engineering. Technology \& Applied Science Research, Vol. 7, N 3, Kwait, 2017.

[15] PANNILA A. S.;JAYANANDA M. K.;SONNADARA D. U. J. Multifractal behavior of wind speed and wind direction. Fractals, 2016, Vol.24, Sri Lanka

[16] RODRIGUES, A. M.; PITA, G. P. A. Caracterização do Escoamento e Fluxo Atmosférico de Calor Latente em Montado de Sobro. Silva Lus , v. 11, n. 2, p. 165184, Lisboa, 2003.

[17] ABBASI A., ANNOR F. O., GIESEN N. V. D.; The effects of small water surfaces on turbulent flow in the atmospheric boundary layer: URANS approach implemented in OpenFOAM, Environmental Modelling \& Software, Volume 101, Pages 268-288, 2018 